Chirurg 2020 $91: 934-942$ https://doi.org/10.1007/s00104-020-01180-0 Online publiziert: 8 . Juni 2020

(c) Der/die Autor(en) 2020

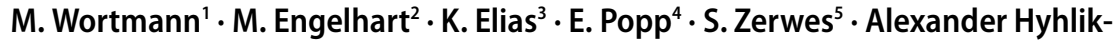
Dürr ${ }^{5}$

'Klinik für Gefäßchirurgie und Endovaskuläre Chirurgie, Universitätsklinikum Heidelberg, Heidelberg, Deutschland

${ }^{2}$ Klinik für Gefäßchirurgie und Endovaskuläre Chirurgie, Bundeswehrkrankenhaus Ulm, Ulm, Deutschland ${ }^{3}$ Abteilung für Gefäßchirurgie, Bundeswehrkrankenhaus Westerstede/Ammerland Klinik, Westerstede, Deutschland

${ }^{4}$ Sektion Notfallmedizin, Klinik für Anästhesiologie, Universitätsklinikum Heidelberg, Heidelberg, Deutschland

${ }^{5}$ Gefäßchirurgie und endovaskuläre Chirurgie, Medizinische Fakultät, Universitätsklinikum Augsburg, Augsburg, Deutschland

\title{
„Resuscitative endovascular balloon occlusion of the aorta" (REBOA)
}

\section{Aktuelles zu Material, Indikationen und Grenzen: ein Überblick}

Vergessenheit geraten [1]. Durch die zunehmende Verbreitung endovaskulärer Techniken in der Versorgung polytraumatisierter Patienten erlebt das Verfahren momentan jedoch eine Renaissance und stellt zurzeit ein diskutiertes Thema in der Notfallmedizin dar. Dies wird nicht nur durch vermehrte Sitzungen zu REBOA auf traumatologischen und notfallmedizinischen Fachtagungen deutlich. Inzwischen gibt es sogar internationale Kongresse, die sich nur mit der Anwendung endovaskulärer Verfahren bei der Traumaversorgung beschäftigen (z.B. Endovascular Resuscitation and Trauma Management (EVTM) Symposium). Auch die rapide steigende Anzahl von mehr als 130 PubMed gelisteten Publikationen allein in den Jahren 2018 und 2019 zeigt das Interesse an dieser wiederentdeckten Technik.

Gerade bei traumatologischen Patienten mit einem hämorrhagischen Schock aufgrund einer Blutung des Abdomens oder des Beckens scheint der Einsatz eines Ballons zur Okklusion der Aorta zur Blutungskontrolle und Aufrechterhaltung der lebensnotwendigen zerebralen und kardialen Perfusion ein vielversprechendes Verfahren zur Verbesserung der Überlebensraten und der Behandlungsergebnisse $\mathrm{zu}$ sein. Zudem stellt es gerade bei Patienten in extremis im Vergleich zur offen chirurgischen Aortenklemmung, die bei traumatologischen Patienten nur mit einer Überlebensrate von $10 \%$ einhergeht $[2,3]$, einen Eingriff mit bedeutend geringerer Invasivität dar.

Über die Anwendung von REBOA bei traumatologischen Blutungen des Körperstammes und stammnahen Blutungen hinaus wird die endovaskuläre Ballonblockade der Aorta auch bei nichttraumatologische Hämorrhagien wie z. B. bei gynäkologischen Blutungen, gastrointestinalen Blutungen und postoperativen Nachblutungen nach abdominellen Eingriffen diskutiert [4, 5]. Nicht zuletzt ist auch eine Anwendung im Rahmen nichttraumatisch bedingter Herz-Kreislauf-Stillstände denkbar.

Durch dieses erweiterte Indikationsspektrum ist REBOA inzwischen nicht nur für Notfallmediziner und Traumatologen von Interesse. Die Technik, das Indikationsspektrum, aber auch Gefahren durch die Anwendung sollten allen chirurgisch tätigen Kolleginnen und Kollegen geläufig sein.
Die Autoren M. Wortmann und M. Engelhart sind gleichberechtigte Erstautoren. 


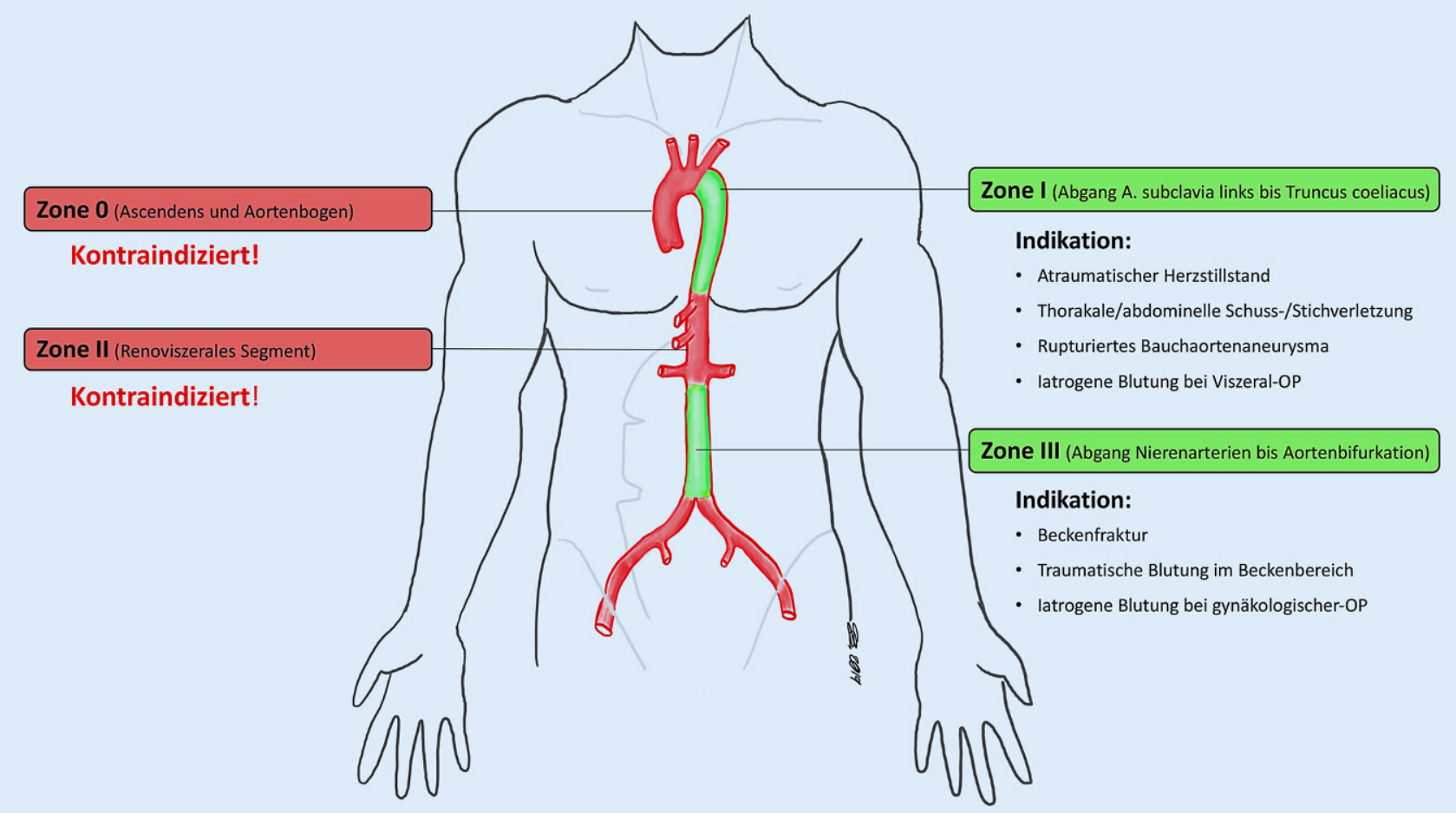

Abb. 1 ॥ REBOA(,resuscitative endovascular balloon occlusion of the aorta“)-Landezone und dazugehörige Indikationen.

OPOperation

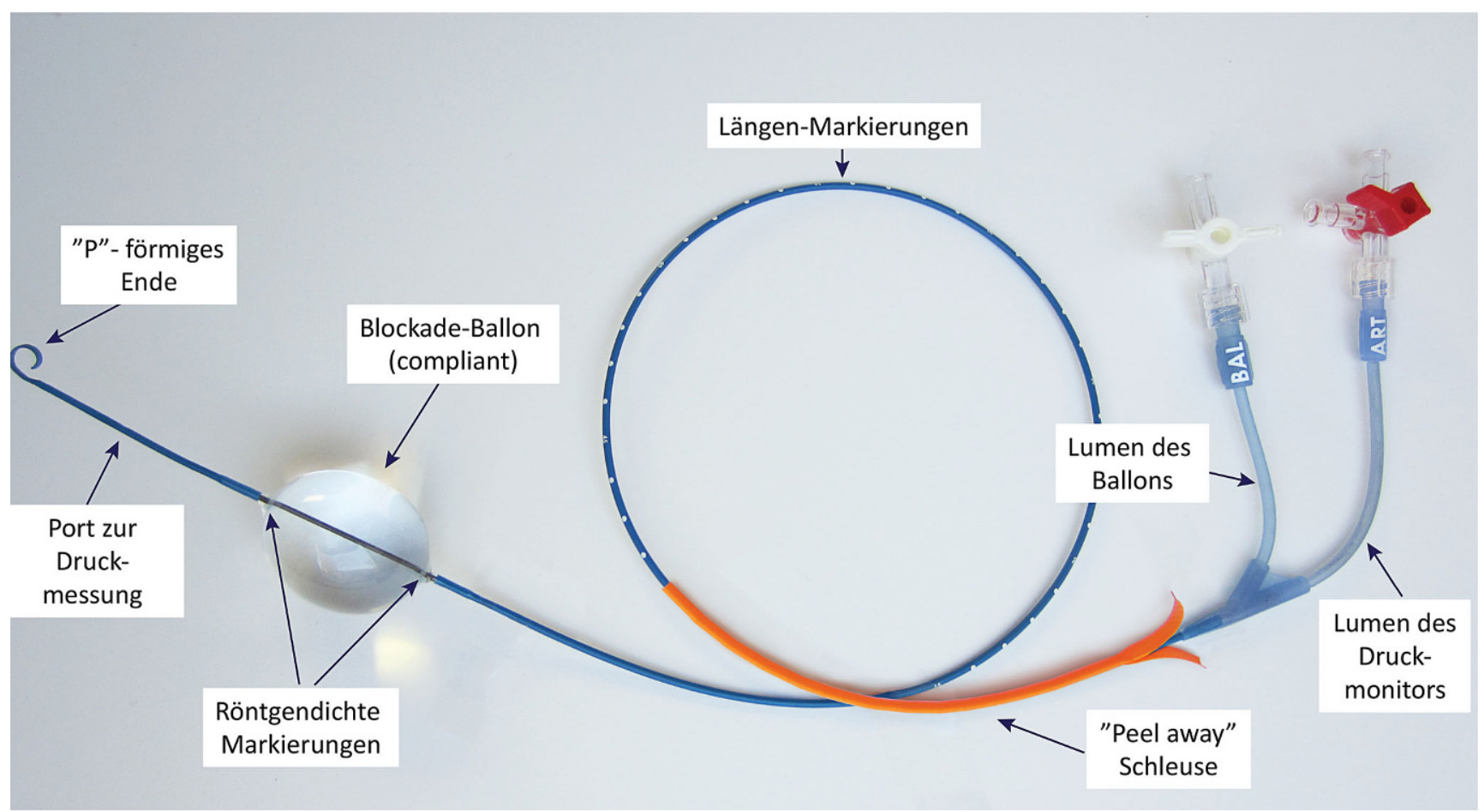

Abb. $2 \triangle \mathrm{ER}-R E B O A^{\mathrm{TM}}$ (Prytime Medical, USA) 
Im folgenden Übersichtsartikel soll die Technik von REBOA einschließlich der verfügbaren und benötigten Materialien vorgestellt werden, die möglichen Einsatzgebiete aufgezeigt, aber auch mögliche Komplikationen durch den Einsatz von REBOA beschrieben werden, um eine neutrale Beurteilung der Methode zu ermöglichen.

\section{Indikationen}

\section{Traumatisch bedingte Blutungen}

REBOA stellt eine weniger invasive, endovaskuläre Alternative zur Notfallthorakotomie mit supradiaphragmaler Klemmung der Aorta bei nichtkomprimierbaren Blutungen im Bauch- und Beckenraum dar. Während tierexperimentelle Studien die Überlegenheit der Ballonokklusion mit zuverlässigem Anstieg des zentralen Perfusionsdrucks bei gleichzeitig geringerem physiologischem Stress belegen konnten [6], ist die Evidenz hinsichtlich eines besseren Behandlungsergebnisses bei Traumapatienten nach wie vor schwach. Dies liegt zum einen an der hohen Letalität der meist polytraumatisierten Patienten, zum anderen an dem selbst in großen Traumazentren kleinen Fallzahlen mit großer Heterogenität des Verletzungsmusters.

Ersten vielversprechenden Fallserien aus dem zivilen [7] und militärischen Bereich [8] zu REBOA bei Trauma folgten widersprüchliche größere Serien. Erste Auswertungen des „Aortic Occlusion for Resuscitation in Trauma and Acute Care Surgery-Registry“, in dem Daten zu REBOA und zur Notfallthorakotomie aus großen US-Traumazentren verglichen werden, belegen eine Überlegenheit von REBOA [9, 10]. Die jüngste Publikation dieser Daten umfasste 285 Patienten (Notfallthorakotomie $71 \%$ vs. REBOA 29\%) und zeigt bei vergleichbarer Verletzungsschwere einen signifikanten Überlebensvorteil für die REBOAGruppe (gesamt Überlebensrate: Notfallthorakotomie $44 \%$ vs. REBOA $63 \%$ ), vor allem bei Patienten ohne kardiopulmonaler Reanimation (Überlebensrate bis Entlassung: Notfallthorakotomie $0 \%$ vs. REBOA $44 \%$; [10]). Kritiker bemängeln

Chirurg 2020 - 91:934-942 https://doi.org/10.1007/s00104-020-01180-0

(c) Der/die Autor(en) 2020

M. Wortmann · M. Engelhart · K. Elias · E. Popp · S. Zerwes · A. Hyhlik-Dürr

"Resuscitative endovascular balloon occlusion of the aorta" (REBOA). Aktuelles zu Material, Indikationen und Grenzen: ein Überblick

\section{Zusammenfassung}

Hintergrund. „Resuscitative endovascular balloon occlusion of the aorta" (REBOA) stellt ein endovaskuläres Verfahren dar, bei dem ein Blockballon in die Aorta eingeführt wird, um eine distal des Ballons gelegene Blutung zu verringern und gleichzeitig die kardiale und zerebrale Oxygenierung zu verbessern.

Ziel der Arbeit. Vorstellung der REBOATechnik, der möglichen Indikationen, der benötigen Materialien und der möglichen Komplikationen des Verfahrens.

Material und Methoden. Nichtsystematischer Übersichtsartikel über die aktuelle Literatur. Ergebnisse. REBOA stellt gerade bei traumatisch bedingten Blutungen und rupturierten Aortenaneurysmen ein mögliches additives Verfahren zur hämodynamischen Stabilisierung dar. Die Komplikationsrate des Verfahrens liegt bei ungefähr $5 \%$, wobei Zugangskomplikationen im Vordergrund stehen, jedoch auch letale Komplikationen möglich sind.
Diskussion. Eine aortale Ballonblockade wird bei der Versorgung rupturierter Aortenaneurysmen standardmäßig eingesetzt. Es gibt wachsende Evidenz, dass REBOA bei der Versorgung polytraumatisierter Patienten mit einem hämorrhagischen Schock aufgrund einer abdominellen oder viszeralen Blutung eine vergleichsweise minimal-invasive Alternative zur offen chirurgischen Aortenklemmung mittels Thorakotomie darstellt. Mit der Entwicklung neuer Ballonkatheter, die ohne Führungsdraht und mit geringeren Schleusendurchmessern auskommen, wird auch ein Einsatz bei anderen Krankheitsbildern wie postoperativen abdominellen Nachblutungen, gynäkologischen Blutungen oder als additives Verfahren bei der kardiopulmonalen Reanimation diskutiert.

Schlüsselwörter

REBOA - Ballon Blockade Aorta - REBOA

Technik · REBOA Komplikationen · Polytrauma

\section{Resuscitative endovascular balloon occlusion of the aorta (REBOA). Current aspects of material, indications and limits: an overview}

\section{Abstract}

Background. Resuscitative endovascular balloon occlusion of the aorta (REBOA) describes an endovascular procedure in which a blocking balloon is introduced into the aorta to reduce bleeding situated distal to the balloon and simultaneously to improve cardiac and cerebral oxygenation.

Objective. Presentation of the REBOA technique, the possible indications, the required material and possible complications of the procedure.

Material and methods. Non-systematic review of the currently available literature. Results. The REBOA procedure is an adjunct to achieve hemodynamic stabilization in patients with traumatic hemorrhage and ruptured aortic aneurysms. The complication rate of the procedure is approximately $5 \%$, whereby access complications are the most common; however, fatal complications are also possible.
Conclusion. A balloon block of the aorta is well established in the treatment of ruptured aortic aneurysms. There is growing evidence that REBOA is a minimally invasive alternative to open surgical cross-clamping of the aorta by thoracotomy for the treatment of patients with polytrauma and hemorrhagic shock due to abdominal or visceral bleeding. Due to the development of new balloon catheters, which can be placed without stiff guidewires and require smaller sheath diameters, REBOA is also discussed for treatment of postoperative abdominal or gynecological bleeding or as a possible adjunct to cardiopulmonary resuscitation for nontraumatic cardiac arrest.

Keywords

REBOA - Ballon aortic occlusion - REBOA technique $\cdot$ REBOA complications $\cdot$ Polytrauma 


\begin{tabular}{|c|c|c|c|c|}
\hline Produkt & Ballondurchmesser & $\begin{array}{l}\text { Schleusen- } \\
\text { größe }\end{array}$ & $\begin{array}{l}\text { Führungs- } \\
\text { draht für } \\
\text { Katheter }\end{array}$ & $\begin{array}{l}\text { Operativer } \\
\text { Gefäß- } \\
\text { verschluss }\end{array}$ \\
\hline $\begin{array}{l}\text { Coda }^{\circledR} \text { Balloon } \\
\text { (Cook Medical, } \\
\text { Boomington, USA) }\end{array}$ & Max. $32 \mathrm{~mm}$ & $\begin{array}{l}12 \mathrm{Fr} \\
45-60 \mathrm{~cm}\end{array}$ & $\mathrm{Ja}$ & $\mathrm{Ja}$ \\
\hline $\begin{array}{l}\text { Reliant }^{\mathrm{TM}} \\
\text { (Medtronic, } \\
\text { Minneapolis, USA) }\end{array}$ & $10-46 \mathrm{~mm}$ & $\begin{array}{l}12 \mathrm{Fr} \\
45-60 \mathrm{~cm}\end{array}$ & $\mathrm{Ja}$ & $\mathrm{Ja}$ \\
\hline $\begin{array}{l}\text { Rescue Balloon }{ }^{\circledR} \\
\text { (Tokai Medical, } \\
\text { Sarayashiki Taraga } \\
\text { Kasugai-city, Japan) }\end{array}$ & $16-40 \mathrm{~mm}$ & $7 \mathrm{Fr}$ & $\mathrm{Ja}$ & Nein \\
\hline $\begin{array}{l}\text { ER-REBOA }^{\text {TM }} \\
\text { (Prytime Medical, }^{\text {Boerne TX, USA) }}\end{array}$ & - & $7 \mathrm{Fr}$ & Nein & Nein \\
\hline $\begin{array}{l}\text { Reboa Balloon } \mathrm{Kit}^{\mathrm{TM}} \\
\text { (Reboa Medical AS, } \\
\text { Norway) }\end{array}$ & $\begin{array}{l}15-30 \mathrm{~mm} \text { (Zone III) } \\
20-30 \mathrm{~mm} \text { (Zone I) }\end{array}$ & $\begin{array}{l}6 / 7 \mathrm{Fr} \\
8-15 \mathrm{~cm}\end{array}$ & $\mathrm{Ja}$ & Nein \\
\hline
\end{tabular}

jedoch den direkten Vergleich beider Verfahren, da REBOA und Notfallthorakotomie nicht notwendigerweise die gleichen Indikationen hätten [11]. In einer retrospektiven Fall-Kontroll-Studie, bei der 140 Patienten, die REBOA erhalten hatten, 280 Patienten ohne REBOA als Vergleichsgruppe zugeordnet wurden, war kein Unterschied in Bezug auf die Menge der verabreichten Blutprodukte oder die Länge des Krankenhausaufenthaltes nachweisbar. Allerdings fanden Joseph et al. eine höhere Mortalität in der REBOA-Gruppe (36\% vs. $19 \%$ ), einehöhere Rate an akutem Nierenversagen (11\% vs. $3 \%)$ und Amputationen der unteren Extremitäten (4\% vs. $1 \%$; [11]). Die Autoren führten diese Unterlegenheit auf den Zeitverlust bis zur Operation und die viszerorenale Ischämiezeit in der REBOAGruppe zurück.

In einer Metaanalyse zeigte sich ein signifikanter Überlebensvorteil bei traumatologischen Patienten durch die Verwendung von REBOA [12]. Bei polytraumatisierten Patienten mit hämorrhagischem Schock konnte durch den Einsatz von REBOA der zentrale systolische Blutdruck um knapp $80 \mathrm{mmHg}$ angehoben werden, was die Effektivität des Verfahrens unterstreicht. Die Komplikationsrate durch das Verfahren lag in dieser Studie bei $5 \%$.

Zusammenfassend gibt es also noch wenig Evidenz bezüglich der Überlegenheit von REBOA im Rahmen der Versor- gung polytraumatisierter Patienten, wobei die bislang publizierten Daten einen guten Effekt in Bezug auf die hämodynamische Stabilisierung bei akzeptabler Komplikationsrate zeigen. Somit erscheint REBOA durchaus als valide Alternative zur Notfallthorakotomie. Da jedoch auch negative Daten bezüglich des Behandlungsergebnisses vorliegen, sind weitere Studien notwendig, um die Indikationsstellung zu verbessern und somit Patienten zu identifizieren, die von dem Verfahren profitieren können.

In der aktuellen S3-Leitlinie Polytrau$\mathrm{ma} /$ Schwerverletztenbehandlung in der aktualisierten Fassung von 2016 wird REBOA als mögliche Maßnahme zur Behandlung kreislaufinstabiler Patienten in extremis aufgeführt, allerdings mit niedrigem Empfehlungsgrad [13].

Dies deckt sich mit internationalen Empfehlungen, z. B. des American College of Surgeons Committee on Trauma (ACS COT) und des American College of Emergency Physicians (ACEP; [14]).

Basierend auf den Daten des TARN (Trauma Audit and Research Network) in England und Wales errechneten Barnard et al. eine Rate von 4 potenziellen REBOA-Patienten pro Jahr in Major Trauma Centres (MTC) und von 9 Patienten pro Jahr in den größten 10 Traumazentren [15]. In kleineren TraumaUnits ist statistisch sogar nur alle 3 Jahre mit einem REBOA-Patienten zu rechnen. In einer retrospektiven Analyse des
TraumaRegister $\mathrm{DGU}^{\circledR}$ zeigten sich für Deutschland ähnlich niedrige Raten von einem Patienten pro Jahr in überregionalen, 0,1 Patienten pro Jahr in regionalen und 0,01 Patienten pro Jahr in lokalen Traumazentren [16]. Diese niedrigen Inzidenzen wiederum stellen die Implementierung von REBOA inklusive der Vorhaltung des notwendigen Materials und die Schulung des Personals infrage, falls diese Strukturen nicht sowieso bereits lokal vorgehalten werden, z. B. für die Versorgung rupturierter Aortenaneurysmen.

\section{Rupturiertes Aortenaneurysma}

Bei der Versorgung rupturierter Aortenaneurysmen ist eine endovaskuläre Ballonblockade im Sinne eines REBOAManövers ein etablierter Teil der Versorgungsstrategie bei hämodynamisch instabilen Patienten [17]. Gerade bei der endovaskulären Versorgung bietet sich das Einbringen eines Blockballons über die in den Leisten einliegenden Schleusen an. Durch einen intraoperativen Wechsel des Blockballons kann eine komplette endovaskuläre Aneurysmaausschaltung unter Ballonblockade der Aorta erfolgen [18]. Aber auch bei der offen chirurgischen Therapie eines rupturierten Aortenaneurysmas ist die Platzierung eines aortalen Blockballons ein probates Mittel zur hämodynamischen Stabilisierung des Patienten bis zur offen chirurgischen Aortenklemmung. Da in Aortenzentren das für das Manöver benötigte Material und die Expertise vorhanden sind, bietet sich hier auch eine breitere Anwendung des REBOA-Manövers im Rahmen anderer Akutsituationen an.

\section{Peripartale gynäkologische Blutungen}

Auch peripartale gynäkologische Blutungen, z. B. bei Placente previa, stellen eine mögliche Indikation für REBOA dar. Hierbei könnte nicht nur der Blutverlust während der Operation reduziert werden, sondern auch die Rate an Hysterektomien verringert werden. Einschränkend muss erwähnt werden, dass für diese Indikation die Datenlage noch ge- 
Tab. 2 Gefäßassoziierte Komplikationen bei REBOA

\begin{tabular}{|c|c|c|c|}
\hline Komplikation & Autor, Jahr & Rate & \\
\hline \multicolumn{4}{|c|}{ Gefäßassoziierte Komplikationen } \\
\hline \multirow{4}{*}{$\begin{array}{l}\text { Aortoiliakale Verletzung } \\
\text { (z. B. Intimaläsion, } \\
\text { Dissektion, Thrombose, } \\
\text { Ruptur) }\end{array}$} & Pieper et al., 2018 [32] & \multirow[t]{4}{*}{-} & \\
\hline & Brenner et al., 2018 [14] & & \\
\hline & Morrison et al., 2016 [33] & & \\
\hline & Davidson et al., 2018 [34] & & \\
\hline \multirow[t]{3}{*}{ Pseudoaneurysma } & Sadek et al., 2016 [35] & \multirow{3}{*}{$\begin{array}{l}2,2-6,5 \% \\
\text { DuBose }\end{array}$} & \\
\hline & Conti et al., 2017 [36] & & \\
\hline & DuBose et al., 2016 [9] & & \\
\hline \multirow[t]{8}{*}{ Thrombembolie, Ischämie } & DuBose et al., 2016 [9] & \multirow{8}{*}{$\begin{array}{l}0,5-14,2 \% \\
\text { Morrison, } \\
\text { DuBose, Saito, } \\
\text { Sadeghi }\end{array}$} & \\
\hline & Pieper et al., 2018 [32] & & \\
\hline & Davidson et al., 2018 [34] & & \\
\hline & Gamberini et al., 2017 [37] & & \\
\hline & Martinelli et al., 2010 [38] & & \\
\hline & Morrison et al., 2016 [33] & & \\
\hline & Saito et al., 2015 [39] & & \\
\hline & Sadeghi et al., 2018 [40] & & \\
\hline \multirow[t]{5}{*}{ Majoramputation } & Joseph et al., 2019 [11] & \multirow{5}{*}{$\begin{array}{l}3,6-21,3 \% \\
\text { Joseph, Saito }\end{array}$} & \\
\hline & Saito et al., 2015 [39] & & \\
\hline & Sadek et al., 2016 [35] & & \\
\hline & Andres et al., 2016 [41] & & \\
\hline & Gamberini et al., 2017 [37] & & \\
\hline \multicolumn{4}{|l|}{ Technische Probleme } \\
\hline \multirow[t]{2}{*}{ Ballonmigration } & DuBose et al., 2016 [9] & \multirow{2}{*}{$\begin{array}{l}0,2-4 \% \\
\text { DuBose, } \\
\text { Sadeghi }\end{array}$} & \multirow{8}{*}{$\begin{array}{l}0,8 \% \\
\text { (Mortalität) } \\
\text { Morrison }\end{array}$} \\
\hline & Sadeghi et al., 2018 [40] & & \\
\hline \multirow[t]{4}{*}{ Ballonruptur } & Hörer, 2016 [42] & \multirow{4}{*}{$\begin{array}{l}0,1-3 \% \\
\text { Martinelli, } \\
\text { Sadeghi }\end{array}$} & \\
\hline & Martinelli et al., 2010 [38] & & \\
\hline & Brenner et al., 2018 [14] & & \\
\hline & Sadeghi et al., 2018 [40] & & \\
\hline \multirow{2}{*}{$\begin{array}{l}\text { Einführung, } \\
\text { Katheterplatzierung }\end{array}$} & Davidson et al., 2018 [34] & \multirow[t]{2}{*}{-} & \\
\hline & Gamberini et al., 2017 [37] & & \\
\hline \multicolumn{4}{|l|}{ Sonstige Komplikationen } \\
\hline \multirow[t]{6}{*}{ Akutes Nierenversagen } & Joseph et al., 2019 [11] & \multirow{6}{*}{$\begin{array}{l}10,7-35,7 \% \\
\text { Saito, Joseph, } \\
\text { Pieper }\end{array}$} & \\
\hline & Pieper et al., 2018 [32] & & \\
\hline & Morrison et al., 2016 [33] & & \\
\hline & Saito et al., 2015 [39] & & \\
\hline & Conti et al., 2017 [36] & & \\
\hline & Davidson et al., 2018 [34] & & \\
\hline Spinale Ischämie & Brenner et al., 2018 [14] & - & \\
\hline \multirow[t]{2}{*}{ Zerebrale Blutung } & Gamberini et al., 2017 [37] & \multirow{2}{*}{$\begin{array}{l}0,1 \% \\
\text { Gamberini }\end{array}$} & \\
\hline & Uchino et al., 2016 [43] & & \\
\hline Schwere Rhabdomyolyse & Pieper et al., 2018 [32] & 46,9\% Pieper & \\
\hline \multirow[t]{3}{*}{ Kompartmentsyndrom } & Wasicek et al., 2018 [44] & \multirow{3}{*}{$\begin{array}{l}\text { 0,7-15\% } \\
\text { Joseph, } \\
\text { Sadeghi, } \\
\text { Wasicek }\end{array}$} & \\
\hline & Sadeghi et al., 2018 [40] & & \\
\hline & Joseph et al., 2019 [11] & & \\
\hline
\end{tabular}

ringer ist als für die Anwendung von REBOA bei traumatologischen Patienten oder im Rahmen der Versorgung rupturierter Aortenaneurysmen. In einer exemplarischen Fallserie von $15 \mathrm{~Pa}$ tientinnen, die im Rahmen der geplanten Sectio bei Placenta previa REBOA erhalten hatten, war die Notwendigkeit zur Hysterektomie und die Menge an transfundierten Blutprodukten im Vergleich zur Kontrollgruppe deutlich reduziert [19]. In dem bereits erwähnten systematischen Review aus dem Jahr 2018 sind bereits 5 Einzelfallbericht respektive kleinere Fallserien eingeschlossen [12]. Gerade bei einer geplanten Sectio mit erwartetem größerem Blutverlust scheint die früh elektive Anlage einer arteriellen Schleuse in der Leiste unter duplexsonographischer Kontrolle vor Beginn des Eingriffs ein probates Mittel, um im Falle einer während der Operation eintretenden starken Blutung direkt einen aortalen Blockballon in die infrarenale Aorta vorbringen zu können. Dabei erscheinen vor allem spezielle REBOA-Katheter mit geringer Schleusengröße vielversprechend, da hierdurch das Zugangstrauma minimiert wird (siehe Technik und Material).

\section{Nichttraumatische abdominelle oder pelvine Blutungen}

Auch nichttraumatische Blutungen im Bereich des Abdomens oder Beckens können eine Indikation für REBOA darstellen. Hierzu liegen ebenfalls bereits verschiedene Einzelfallberichte sowie kleinere Fallserien vor, die den Einsatz einer aortalen Ballonblockade bei oberen gastrointestinalen Blutungen [20], rupturierten Viszeralarterienaneurysmata [21] oder Nachblutungen im Rahmen viszeralchirurgischer Operationen [22], insbesondere Pankreasoperationen [23], beschreiben. Vergleichbar mit der Verwendung bei traumatisch bedingten Blutungen bewirkt REBOA bei dieser Form der Blutungen im Durchschnitt einen Blutdruckanstieg von ca. $50 \mathrm{~mm} \mathrm{Hg} \mathrm{[24].}$

\section{Nichttraumatischer Herz-Kreislauf-} Stillstand

Auch beim nichttraumatischen HerzKreislauf-Stillstand ohne Vorliegen ei- 


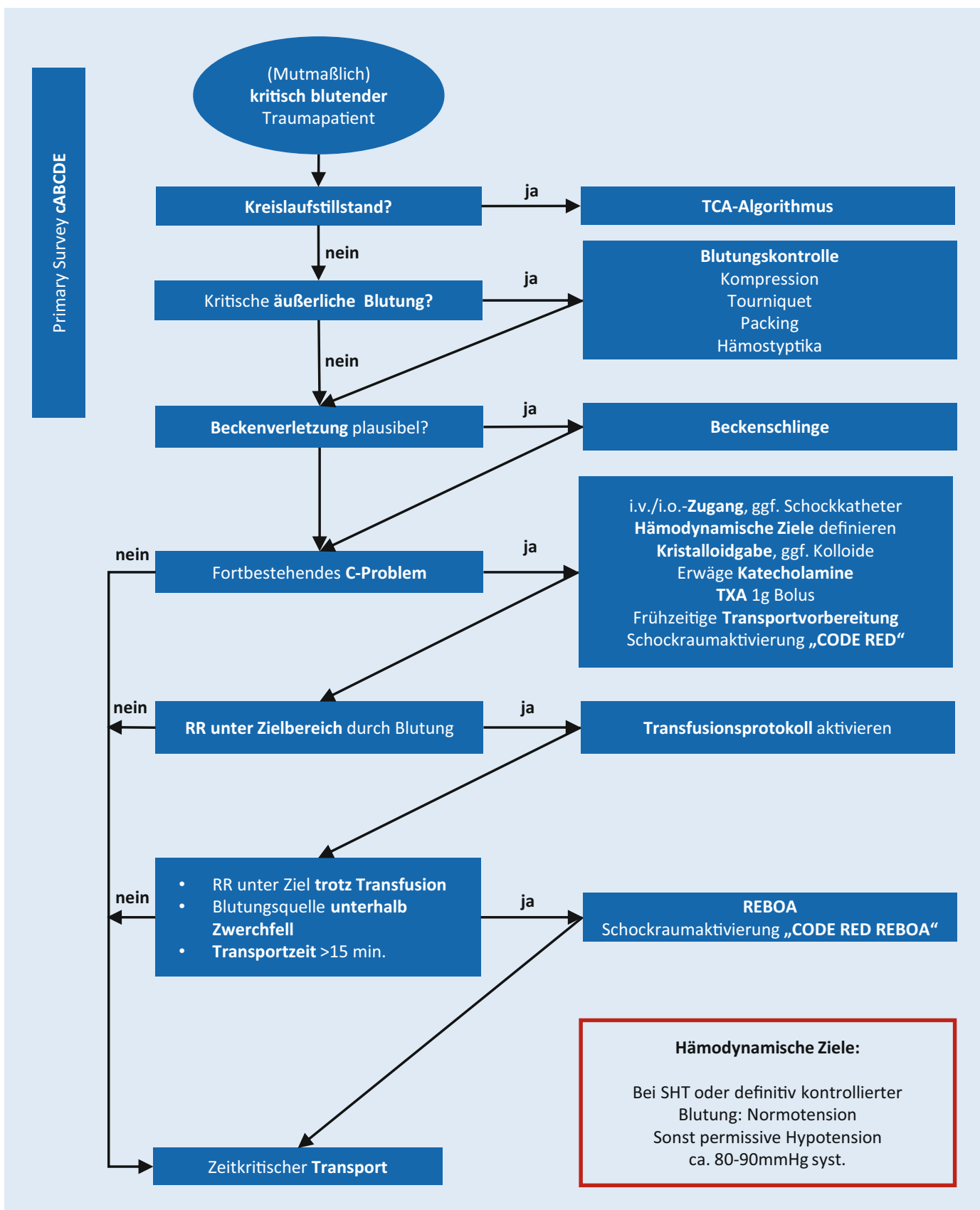

Abb. $3 \triangleleft$ Algorithmus zur präklinischen Behandlung kritisch verletzter Patienten, wie er auf dem "Medical Intervention Car (MIC)" des UKHD zum Einsatz kommt. Dieser inkludiert in eskalierender Weise die Transfusion von Erythrozytenkonzentraten und die Anwendung von REBOA, falls die hämodynamischen Ziele konventionell nicht zu erreichen sind und die nächste geeignete Klinik mehr als 15 min entfernt ist. $R R$ systolischer Blutdruck, TCA-Algorithmus Algorithmus zum traumatischen HerzKreislauf-Stillstand nach Empfehlungen des ERC 2015, i.v./i.o.-Zugang intravenöser/intraossärer Zugang, Schockkatheter großlumiger (12F) zentralvenöser Katheter mit $3 \mathrm{~lm}$. (Mit freundlicher Genehmigung der Mitglieder der AG MIC der Klinik für Anästhesiologie des UKHD)

ner Blutung kann durch REBOA eine Zentralisierung des Kreislaufes und somit eine Verbesserung der kardialen und zerebralen Perfusion und Oxygenierung während der Reanimation erreicht werden. Somit wird auch in diesem Kontext der Einsatz von REBOA diskutiert, ohne dass diesbezüglich außerhalb von Einzelfallberichten und Fallserien belastbare Evidenz vorliegt $[25,26]$.

\section{Technik und Material}

Die Idee der aortalen Ballonokklusion bei schwerer traumatischer Blutung wurde bereits in den 50er-Jahren des letzten Jahrhunderts von Colonel Hughes vorgestellt [1]. Wegen der unbefriedigenden Ergebnisse wurde das Verfahren jedoch zunächst nicht weiterverfolgt bis Fortschritte in der endovaskulären Technik und verbesserte Materialien den Einsatz der Ballonokklusion zunächst beim rupturierten Bauchaortenaneurysma recht- fertigten. Mit REBOA im engeren Sinne wurden die Indikationen auf traumatische und zunehmend auch nichttraumatische Blutungen in Abdomen und Becken erweitert.

Zunächst kamen aortale Okklusionsballons, wie sie aus der Gefäßchirurgie bekannt waren, für REBOA zum Einsatz. Weit verbreitet waren der Coda Balloon ${ }^{\circledR}$ (Fa. Cook Medical, USA) und der Reliant Balloon ${ }^{\circledR}$ (Fa. Medtronic, USA; • Tab. 1). Beide mussten über einen Führungsdraht unter Durchleuchtung vorgeschoben 
werden und benötigten eine lange $60 \mathrm{~cm}$ (Aorta descendens, REBOA-Zone_I) oder kurze $45 \mathrm{~cm}$ (infrarenale Aorta, REBOA-Zone-III) 14-Fr- bzw. 12-FrSchleuse zur Stabilisierung in der Aorta. Neben den praktischen Problemen mit Platzierung der 7-Fr-Einführschleuse in Seldinger-Technik, Wechsel auf eine 12bis 14-Fr-Schleuse und der Handhabung langer, steifer Führungsdrähte, welche für den nichtendovaskulär geübten Traumachirurgen eine ungewohnte Herausforderung darstellten und Zeit kosteten, provozierten vor allem die kaliberstarken Schleusen Komplikationen wie großes Zugangstrauma in der Leistenarterie mit Nachblutungen und Pseudoaneurysmata sowie Thrombosen in den ipsilateralen Becken- und Beinarterien bis hin zur sekundären Majoramputation bei akuter Extremitätenischämie [27].

Neue Entwicklungen spezieller Ballonsysteme für REBOA konzentrierten sich daher auf eine Vereinfachung der Handhabung und einen Verzicht auf kaliberstarke Schleusen. Durch den Verzicht auf eine größere Schleuse werden hierbei nicht nur die Komplikationen, sondern auch die Zeit bis zur Inflation des Ballons in der Aorta deutlich reduziert [28]. Der Rescue Balloon ${ }^{\circledR}$ (Tokai Medical, Japan) kommt mit einer kurzen $(8-15 \mathrm{~cm}) 7$-Fr-Einführschleuse aus und stabilisiert sich selbst in der Aorta. Die Platzierung erfolgt jedoch nach wie vor unter Durchleuchtung über einen Führungsdraht. Das Gleiche gilt für den relativ neuen Ballon eines norwegischen Herstellers (Reboa Medical AS, Norwegen), welcher in einem praktischen Reboa Balloon $\mathrm{Kit}^{\mathrm{T} \mathrm{si}}$ einschließlich aller benötigten Materialen geliefert wird.

Gänzlich ohne Führungsdraht für den Katheter und notfalls auch ohne Durchleuchtung kommt der ER-REBOA ${ }^{\mathrm{Tm}}$ (Prytime Medical, USA) aus (• Abb. 2). Dieser Ballon wurde in enger Zusammenarbeit mit den Gefäßchirurgen des US-Militärs entwickelt und soll den sicheren und einfachen Einsatz auch unter ungünstigen, prähospitalen Bedingungen erlauben. Nach Abmessung der notwendigen Katheterlänge mithilfe der Markierungen auf dem Schaft wird der Katheter über eine 7-Fr-Einführschleuse notfalls „blind“ vorgeschoben. Eine atraumati- sche, abgerundete Spitze (P-tip ${ }^{\circledR}$ ), ähnlich der Konfiguration eines Pigtail-Katheters, soll hierbei iatrogene Gefäßverletzung und ein Ausweichen in Seitenäste vermeiden. Ein Kanal mit seitlicher Öffnung erlaubt die Druckmessung und Medikamentenapplikation proximal des insufflierten Ballons. Wie die anderen 7-FrBallons auch ist der Katheter steif genug, um sich selbst in der Aorta zu stabilisieren. In einem Bauchaortenaneurysma können sich die Katheter jedoch nicht an der Wand abstützen und können somit dislozieren. Wie beim Reboa Balloon $\mathrm{Kit}^{\mathrm{Tm}}$ wird auch für den ER-REBOA ${ }^{\mathrm{Tm}}$ ein praktisches Komplettset mit allen Zubehörteilen angeboten. Mit Ausnahme des japanischen Rescue Balloon ${ }^{\circledR}$ sind alle vorgestellten Katheter inzwischen auch für den deutschen Markt zugelassen.

Aktuelle Publikationen zeigen, dass sich die Low-profile-REBOA-Ballons gegenüber den kaliberstarken Kathetern weitgehend durchgesetzt haben [27]. Im militärischen Bereich ist der ERREBOA $^{\mathrm{Ts}}$ wegen seiner vergleichsweisen einfachen Handhabung weit verbreitet. Weitere Entwicklungen gehen z. B. in Richtung einer Erleichterung der Platzierung des Ballons ohne Durchleuchtung [29].

\section{Komplikationen}

Wie jedes endovaskuläre Verfahren birgt REBOA multiple Komplikationsrisiken. Besonders der perkutane Zugang zur A. femoralis communis im Bereich der Leiste ist bei Patienten unter Reanimation oder minimalem Kreislauf oftmals nicht sicher möglich, sodass Zentren mit größeren Fallzahlen eine relevante Anzahl offen chirurgischer Leistenfreilegungen beschrieben, um überhaupt einen Zugang zum Gefäß zu erlangen. Prinzipiell können die Komplikationen in Komplikationen durch die Malperfusion distal des inflatierten Blockballons, in Zugangskomplikationen sowie Komplikationen durch Dislokation des Drahtes und des Blockballons eingeteilt werden [30]. In dem bereits mehrfach zitierten systematischen Review liegt die Komplikationsrate bei ca. 5\% [12]. Dabei sind besonders bei langer Lagezeit der Blockballons arterielle Thrombosen besonders häufig [31]. Somit ist im Rahmen des Verfahrens eine gefäßchirurgische Expertise vor Ort unbedingt notwendig. Erste Ansätze, REBOA nicht nur im klinischen Setting, sondern auch prähospital einzusetzen, bedürfen einer speziellen Ausstattung der Notfallteams sowie einer standardisierten Patientenselektion, wie dies im Rahmen eines Forschungsprojekts für invasive Notfalltechniken schon etabliert wird (•Abb. 3). Eine Zusammenfassung möglicher Gefäßassoziierter Komplikationen zeigt • Tab. 2.

\section{Fazit für die Praxis}

- REBOA bezeichnet die endovaskuläre Ballonblockade der Aorta zur hämodynamischen Stabilisierung bei hämorrhagischem Schock.

- REBOA ist ein etabliertes Verfahren bei der Therapie rupturierter Aortenaneurysmen.

- Die aktuelle Evidenz erlaubt den Einsatz von REBOA bei Polytraumatisierten mit einem hämorrhagischen Schock aufgrund einer abdominellen, pelvinen oder stammnahen Blutung als Alternative zur Notfallthorakotomie.

- Neue, speziell für das Verfahren entwickelte Ballonkatheter kommen mit geringeren Schleusendurchmessern aus oder sind durch den Verzicht auf einen steifen Führungsdraht schneller platzierbar.

- Die Komplikationsrate liegt bei ca. $5 \%$, wobei Zugangskomplikationen im Vordergrund stehen.

- Der Einsatz von REBOA wird auch bei anderen Krankheitsbildern wie gynäkologischen oder postoperativen abdominellen oder pelvinen Nachblutung diskutiert.

- Da auch bereits Komplikationen mit letalen Ausgängen und Fallserien mit einem schlechteren Behandlungsergebnis in der REBOA-Gruppe publiziert sind, sind weitere Studien zur Verbesserung der Patientenauswahl und Indikationsstellung notwendig. 


\section{Korrespondenzadresse}

Prof. Dr. Alexander Hyhlik-Dürr

Gefäßchirurgie und endovaskuläre Chirurgie, Medizinische Fakultät, Universitätsklinikum Augsburg

Stenglinstr. 2, 86156 Augsburg, Deutschland alexander.hyhlik-duerr@uk-augsburg.de

Funding. Open Access funding provided by Projekt DEAL.

\section{Einhaltung ethischer Richtlinien}

Interessenkonflikt. M. Wortmann, M. Engelhart und A. Hyhlik-Dürr geben an, dass kein Interessenkonflikt besteht.

Für diesen Beitrag wurden von den Autoren keine Studien an Menschen oder Tieren durchgeführt. Für die aufgeführten Studien gelten die jeweils dort angegebenen ethischen Richtlinien.

Open Access. Dieser Artikel wird unter der Creative Commons Namensnennung 4.0 International Lizenz veröffentlicht, welche die Nutzung, Vervielfältigung, Bearbeitung, Verbreitung und Wiedergabe in jeglichem Medium und Format erlaubt, sofern Sie den/die ursprünglichen Autor(en) und die Quelle ordnungsgemäß nennen, einen Link zur Creative Commons Lizenz beifügen und angeben, ob Änderungen vorgenommen wurden.

Die in diesem Artikel enthaltenen Bilder und sonstiges Drittmaterial unterliegen ebenfalls der genannten Creative Commons Lizenz, sofern sich aus der Abbildungslegende nichts anderes ergibt. Sofern das betreffende Material nicht unter der genannten Creative Commons Lizenz steht und die betreffende Handlung nicht nach gesetzlichen Vorschriften erlaubt ist, ist für die oben aufgeführten Weiterverwendungen des Materials die Einwilligung des jeweiligen Rechteinhabers einzuholen.

Weitere Details zur Lizenz entnehmen Sie bitte der Lizenzinformation auf http://creativecommons.org/ licenses/by/4.0/deed.de.

\section{Literatur}

1. Hughes CW (1954) Use of an intra-aortic balloon catheter tamponade for controlling intraabdominal hemorrhage in man. Surgery $36: 65-68$

2. Working Group, Ad Hoc Subcommittee on Outcomes, American College of Surgeons. Committee on Trauma. (2001) Practice management guidelines for emergency department thoracotomy. Working group, ad hoc subcommittee on outcomes, American college of surgeons-committee on trauma. J Am Coll Surg 193:303-309

3. Edens JW, Beekley AC, Chung KK et al (2009) Longterm outcomes after combat casualty emergency department thoracotomy. J Am Coll Surg 209:188-197

4. Ordonez CA, Manzano-Nunez R, Parra MW et al (2018) Prophylactic use of resuscitative endovascular balloon occlusion of the aorta in women with abnormal placentation: a systematic review, meta-analysis, and case series. J Trauma Acute Care Surg 84:809-818

5. Brede JR, Lafrenz T, Kruger AJ et al (2019) Resuscitative endovascular balloon occlusion of the aorta (REBOA) in non-traumatic outof-hospital cardiac arrest: evaluation of an educational programme. BMJ Open 9:e27980

6. White JM, Cannon JW, Stannard A et al (2011) Endovascular balloon occlusion of the aorta is superior to resuscitative thoracotomy with aortic clamping in a porcine model of hemorrhagic shock. Surgery 150:400-409

7. Brenner ML, Moore LJ, Dubose JJ et al (2013) A clinical series of resuscitative endovascular balloon occlusion of the aorta for hemorrhage control and resuscitation. JTrauma Acute Care Surg 75:506-511

8. Manley JD, Mitchell BJ, Dubose JJ et al (2017) A modern case series of resuscitative endovascular balloon occlusion of the aorta (REBOA) in an outof-hospital, combat casualty care setting. J Spec OperMed 17:1-8

9. Dubose JJ, Scalea TM, Brenner M et al (2016) The AAST prospective aortic occlusion for resuscitation in trauma and acute care surgery (AORTA) registry: data on contemporary utilization and outcomes of aortic occlusion and resuscitative balloon occlusion of the aorta (REBOA). J Trauma Acute Care Surg 81:409-419

10. Brenner M, Inaba K, Aiolfi A et al (2018) Resuscitative endovascular balloon occlusion of the aorta and resuscitative thoracotomy in select patients with hemorrhagic shock: early results from the American association for the surgery of trauma's aortic occlusion in resuscitation for trauma and acute care surgery registry. J Am Coll Surg 226:730-740

11. Joseph B, Zeeshan M, Sakran JV et al (2019) Nationwide analysis of resuscitative endovascular balloon occlusion of the aorta in civilian trauma. JAMA Surg 154:500-508

12. Borgervan der Burg BLS, van Dongen T, Morrison JJ et al (2018) A systematic review and meta-analysis of the use of resuscitative endovascular balloon occlusion of the aorta in the management of major exsanguination. Eur J Trauma Emerg Surg 44:535-550

13. Deutsche Gesellschaft für Unfallchirurgie (2016) S3-Leitllinie Polytrauma/Schwerverletzten Behandlung (AWMF Register-Nr. 012/019)

14. Brenner M, Bulger EM, Perina DG et al (2018) Joint statement from the American college of surgeons committee on trauma (ACSCOT) and the American college of emergency physicians (ACEP) regarding the clinical use of resuscitative endovascular balloon occlusion of the aorta (REBOA). Trauma Surg Acute Care Open 3:e154

15. Barnard EB, Morrison JJ, Madureira RM et al (2015) Resuscitative endovascular balloon occlusion of the aorta (REBOA): a population based gap analysis of trauma patients in England and Wales. Emerg Med J 32:926-932

16. Kulla $M$, Engelhardt $M$, Holsträter T et al (2018) Do we need REBOA as an adjunct to ER thoracotomy in German trauma centres? A secondary data analysis from the TraumaRegister DGU. Anasth Intensivmed 59:562-573

17. Starnes BW, Quiroga E, Hutter C et al (2010) Management of ruptured abdominal aortic aneurysm in the endovascular era. J Vasc Surg 51:9-17 (discussion 17-18)

18. BerlandTL, VeithFJ, CayneNSetal (2013) Technique of supraceliac balloon control of the aorta during endovascular repair of ruptured abdominal aortic aneurysms. J Vasc Surg 57:272-275

19. Panici PB, Anceschi M, Borgia ML et al (2012) Intraoperative aorta balloon occlusion: fertility preservation in patients with placenta previa accreta/increta. J Matern Fetal Neonatal Med 25:2512-2516

20. Karkos CD, Bruce IA, Lambert ME (2001) Use of the intra-aortic balloon pump to stop gastrointestinal bleeding. Ann Emerg Med 38:328-331

21. Delamare L, Crognier L, Conil JM et al (2015) Treatment of intra-abdominal haemorrhagic shock by resuscitative endovascular balloon occlusion of the aorta (REBOA). Anaesth Crit Care Pain Med 34:53-55

22. Miura F, Takada T, Ochiai T et al (2006) Aortic occlusion balloon catheter technique is useful for uncontrollable massive intraabdominal bleeding after hepato-pancreato-biliary surgery. J Gastrointest Surg 10:519-522

23. Singh G, Nahm CB, Jamieson NB et al (2019) Management of post-pancreatectomy haemorrhage using resuscitative endovascularballoon occlusion of the aorta. Langenbecks Arch Surg 404:253-255

24. Borger van der Burg BLS, Kessel B, Dubose JJ et al (2019) Consensus on resuscitative endovascular balloon occlusion of the aorta: a first consensus paper using a Delphimethod. Injury 50:1186-1191

25. Deakin CD, Barron DJ (1996) Haemodynamic effects of descending aortic occlusion during cardiopulmonary resuscitation. Resuscitation 33:49-52

26. Daley J, Morrison JJ, Sather J et al (2017) The role of resuscitative endovascular balloon occlusion of the aorta (REBOA) as an adjunct to ACLS in non-traumatic cardiac arrest. Am J Emerg Med 35:731-736

27. Bekdache O, Paradis T, Shen YBH et al (2019) Resuscitative endovascular balloon occlusion of the aorta (REBOA): indications: advantages and challenges of implementation in traumatic noncompressible torso hemorrhage. Trauma Surg Acute Care Open 4:e262

28. Romagnoli AN, Teeter W, Wasicek P et al (2018) No wire? No problem: resuscitative endovascular balloon occlusion of the aorta can be performed effectively and more rapidly with a wire-free device. JTrauma Acute Care Surg 85:894-898

29. Wessels LE, Wallace JD, Bowie J et al (2019) Radiofrequency identification of the ER-REBOA confirmation of placement without fluoroscopy. MilMed 184:e285-e289

30. Wortmann M, Elias K, Zerwes S et al (2019) REBOA (resuscitative endovascular balloon occlusion of the aorta) - Brauchen wir das wirklich? Notf Rett Med 2019(22):100-110

31. Lendrum R, Perkins Z, Chana M et al (2019) Pre-hospital resuscitative endovascular balloon occlusion of the aorta (REBOA) for exsanguinating pelvic haemorrhage. Resuscitation 135:6-13

32. Pieper A, Thony F, Brun J et al (2018) Resuscitative endovascular balloon occlusion of the aorta for pelvic blunt trauma and life-threatening hemorrhage: a 20-year experience in a level I trauma center. J Trauma Acute Care Surg 84:449-453

33. Morrison JJ, Galgon RE, Jansen JO et al (2016) A systematic review of the use of resuscitative endovascular balloon occlusion of the aorta in the management of hemorrhagic shock. J Trauma Acute Care Surg 80:324-334

34. Davidson AJ, Russo RM, Reva VA et al (2018) The pitfalls of resuscitative endovascular balloon 


\section{Übersichten}

occlusion of the aorta: risk factors and mitigation strategies. J Trauma Acute Care Surg 84:192-202

35. Sadek S, Lockey DJ, Lendrum RA et al (2016) Resuscitative endovascular balloon occlusion of the aorta (REBOA) in the pre-hospital setting: an additional resuscitation option for uncontrolled catastrophic haemorrhage. Resuscitation 107:135-138

36. Conti BM, Richards JE, Kundi R et al (2017) Resuscitative endovascular balloon occlusion of the aorta and the anesthesiologist: a case report and literature review.A\&ACase Reports 9:154-157

37. Gamberini E, Coccolini F, Tamagnini B et al (2017) Resuscitative endovascular balloon occlusion of the aorta in trauma: a systematic review of the literature. World JEmerg Surg 12:42

38. Martinelli T, Thony F, Declety P et al (2010) Intraaortic balloon occlusion to salvage patients with life-threatening hemorrhagic shocks from pelvic fractures. JTrauma 68:942-948

39. Saito N, Matsumoto H, Yagi Tetal (2015) Evaluation of the safety and feasibility of resuscitative endovascular balloon occlusion of the aorta. J Trauma Acute Care Surg 78:897-903 (discussion 904)

40. Sadeghi M, Nilsson KF, Larzon Tet al (2018) The use of aortic balloon occlusion in traumatic shock: first report from the $A B O$ trauma registry. Eur J Trauma Emerg Surg 44:491-501

41. Andres J, Scott J, Giannoudis PV (2016) Resuscitative endovascular balloon occlusion of the aorta (REBOA): what have we learned? Injury 47:2603-2605
42. Horer TM, Hebron D, Swaid F et al (2016) Aorta balloon occlusion in trauma: three cases demonstrating multidisciplinary approach already on patient's arrival to the emergency room. Cardiovasc Intervent Radiol 39:284-289

43. Uchino $H$, Tamura N, Echigoya R et al (2016) "REBOA" - is it really safe? A case with massive intracranial hemorrhage possibly due to endovascular balloon occlusion of the aorta (REBOA). Am J Case Rep 17:810-813

44. Wasicek PJ, Teeter WA, Yang S et al (2018) Life ove limb: lower extremity ischemia in the setting of resuscitative endovascular balloon occlusion of the aorta (REBOA). Am Surg 84:971-977

\section{Hier steht eine Anzeige.}

\section{Springer}

